\title{
Tamsulosin and Dementia in Old Age: is There Any Relationship?
}

\author{
Rebecca Renata Lapenda do Monte ${ }^{1}$, Karina de Andrade Vidal Costa ${ }^{1}$, Arnaldo Santiago Nunes \\ Junior ${ }^{2}$, Amália Cinthia Meneses Rêgo ${ }^{3}$ and Irami Araújo Filho ${ }^{4 *}$
}

${ }^{1}$ UnP - Potiguar University, Brazil

${ }^{2}$ Department of Urology, UnP - Laureate International Universities, Brazil

${ }^{3}$ UnP - Laureate International Universities, Brazil

${ }^{4}$ Department of Surgery, Federal University of Rio Grande do Norte, Brazil

*Corresponding author: Irami Araújo-Filho, Postgraduate Program in Biotechnology at Potiguar University/ UnP - Laureate International Universities, Department of Surgery, Federal University of Rio Grande do Norte, Brazil.

To Cite This Article: Rebecca Renata Lapenda do Monte, Karina de Andrade Vidal Costa, Arnaldo Santiago Nunes Junior, Amália Cinthia Meneses Rêgo, Irami Araújo Filho. Tamsulosin and Dementia in Old Age: is There Any Relationship?. Am J Biomed Sci \& Res. 2019 - 5(4). AJBSR.MS.ID.000928. DOI: 10.34297/AJBSR.2019.05.000928.

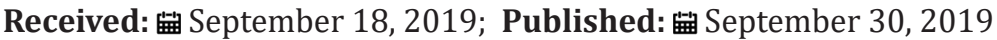

\begin{abstract}
Tamsulosin is used to treat Benign Prostatic Hyperplasia (BPH), prescribed annually to about 12.6 million patients worldwide. It is an alphaadrenergic antagonist that reduces the tone of the prostate smooth muscle involved in the pathophysiology of BPH. By acting on alpha $1 \mathrm{~A}$ receptors, predominant in the prostate, tamsulosin also acts on receptors present in the brain. This study consisted of a literature review aimed at disseminating scientific knowledge about the relationship between the use of tamsulosin and the onset of dementia. Pubmed, Scopus, Scielo, Embase, and Web of Science studies involving dementia in patients using tamsulosin in the last five years were selected. The review showed a risk correlation and a higher incidence of dementia in treated patients. The risk ratio, when compared to other medicines, approached 1.20. In conclusion, it was identified the need for clinical trials with higher sampling power to increase relational significance due to the high prevalence of BPH and the extensive use of tamsulosin in elderly patients with the disease.
\end{abstract}

Keywords: Benign prostatic hyperplasia; Prostatic hyperplasia; Cognitive functions; Tamsulosin; Dementia; Adrenergic alpha-1 receptor antagonists

\section{Introduction}

Benign prostatic hyperplasia (BPH) is a common disease among older men and responsible for several physiological disorders. The healthy prostate weighs approximately $20 \mathrm{~g}$, however, due to the increasing number of individuals with $\mathrm{BPH}$, the average prostate weight at autopsy increases from the age of 50, reaching twice or triple the weight in some cases1. Risk factors associated with BPH are black race, family history of bladder cancer, and prostatitis. Protective factors refer to elevated serum estradiol, testosterone, and anti-inflammatory levels [1]. This condition may be clinically asymptomatic, but $50 \%$ of men aged $\geq 50$ years and $80 \%$ of those $\geq 80$ years have increased urinary frequency, nocturia, urinary urgency, incontinence, hesitation, intermittent flow, bladder emptying effort, slow flow, or the division of urinary flow [2]. Depending on the symptoms and severity of the disease, pharmacological treatment with alpha-adrenergic blockers is indicated, balancing aspects of symptomatic severity and potential side effects of the drug used [2].
Widely widespread, tamsulosin is only instituted in cases in which the patient's quality of life is compromised. Besides, there are many cases of stabilization and attenuation of symptoms, even without therapeutic intervention [2,3]. Tamsulosin has effective selective action, blocks alpha-1A adrenergic receptors and causes mild adverse effects, including orthostatic hypotension. It is an easy-touse medicine that promotes rapid symptomatic improvement and is, therefore, suitable for older patients [3].

Although it has several benefits, its effects in the medium and long term should be considered. Thus, a drug-epidemiological study has pointedly demonstrated the relationship between tamsulosin and the high risk of dementia in this group of patients. One hypothesis is that tamsulosin acts on prostate and brain alpha receptors, thus stimulating cognitive dysfunction, a precursor of dementia [3]. Based on this principle, studies that contribute to elucidate this association brings significant implications of 
high scientific value for the health of the world population that is undergoing a full aging process. Due to the importance of the theme, the present review aimed to evaluate scientifically validated evidence on the use of tamsulosin and its association with the onset of dementia in patients with benign prostatic hyperplasia (BPH) [4].

\section{Methods}

A search was performed in the Pubmed, Scopus, Scielo, Embase, and Web of Science databases, as well as Google Scholar search, considered as gray literature. We selected articles related to the use of tamsulosin and dementia over the past five years and were chosen by combining the following keywords: benign prostatic hyperplasia, prostatic hyperplasia, cognitive functions, tamsulosin, dementia, adrenergic alpha-1 receptor antagonists. There included cohort studies, systematic review, case-control, cross-sectional, case series studies, and randomized controlled trials. The inclusion criteria adopted were human studies, complete articles, of high scientific value, and published in the last five years. The analysis, review, and selection of articles was made by pairs, separately, from the reading of the title and summary of each study, with a third reviewer in case of disagreement between the other two reviewers.

\section{Results and Discussion}

\section{Benign Prostatic Hyperplasia and Dementia}

Benign prostatic hyperplasia (BPH) is defined as the presence of hyperplastic glands during a pathological analysis of prostate tissue. Approximately $60 \%$ of patients have symptoms of moderate to severe BPH. However, new cases at an early stage are detected due to new screening methods [5]. Two mechanisms cause urinary symptoms: excessive growth of prostate tissue that causes obstructions to urinary flow and enlarged prostate smooth muscle cells, resulting in increased resistance to urinary flow [5,6]. Another relevant and current theme concerning the elderly is the increase in the average life expectancy and the high prevalence of Alzheimer's disease and dementia in this age group. These diseases are costly, as are heart disease and cancer, yet effective drug alternatives are scarce [6]. For this reason, it is necessary to understand the pathogenesis of dementia and possible avoidable etiological causes.

\section{Association of Dementia and Tamsulosin}

Duan et al. conducted the first study on the association between Tamsulosin use and the development of dementia in elderly patients with BPH [6]. It was a retrospective cohort study that looked at US Medicare beneficiaries older than 66 years and diagnosed with BPH within six years [7]. Related groups with the same eligibility criteria were used: the first including patients with BPH who did not use any medication, the second by patients using other $\alpha 1$ adrenoreceptor antagonists (doxazosin, terazosin or alfuzosin) and the third group involving patients using $5 \alpha$-reductase inhibitors, i.e., dutasteride, and finasteride [6-8]. All patients were followed for the following outcomes: dementia, exclusion (death, initiation of a different HPB medication, or loss of Medicare coverage) and study termination [7]. The final study sample included: HPB/tamsulosin group ( $\mathrm{n}=253,136) ;$ HPB ( $\mathrm{n}=180,926)$; HPB/doxazosin $(\mathrm{n}=$ 28,581); HPB/terazosin ( $n=23,858) ;$ HPB/alfuzosin ( $n=17,934)$; HPB/dutasteride ( $\mathrm{n}=34,027)$; and HPB/finasteride ( $\mathrm{n}=38,767)$. The average follow-up period was 19.8 months [7]. According to Duan et al., tamsulosin did not show greater efficacy and tolerability than other common $\alpha 1$ receptor antagonists, despite having greater selectivity [3]. As a result, it was observed that the tamsulosin group had a higher gross incidence of dementia, being 38.8/1000 patients/year compared with 24.1-32.8 / 1000 patients/year in the other comparative groups [8]. In addition, these patients scored higher on the Charlson Comorbidity Index and were more likely to have cerebrovascular disease, peripheral vascular disease, congestive heart failure, myocardial infarction, delirium, traumatic brain injury, Parkinson's disease, and depression; requiring greater assistance from health services [7]. Compared to patients without treatment for $\mathrm{BPH}$, the tamsulosin group showed a significantly increased risk of dementia 31.3 versus 25.9 / 1000 person/year. With this in mind, tamsulosin use was significantly associated with an increased risk of dementia when compared with each of the five groups of patients who used alternative medicines for BPH [7]. This first study by Duan et al. is considered in the literature as the teste $\alpha$ and has emerged to determine if there are insights to consider between tamsulosin and dementia. Other criteria, such as $\beta$, have been proposed by Gill et al., who found the number needed to harm (NNP) between drug users and an individual develop the deficit of 186 , so the relationship between developing dementia is relatively small if there is any relationship [6]. Despite poor blood-brain barrier penetration, there is a high affinity for all neurotransmitter receptors ( $\alpha 1$-adrenergic, dopaminergic, and serotonergic) that regulate and modulate essential central nervous system functions such as mood, affect, attention, learning, and memory [9]. Accordingly, a study in humans has shown that there is a reduction in alpha-adrenergic receptors in the prefrontal cortex of elderly patients with associated dementia [4]. One factor that refutes this higher likelihood of associated risk in patients using tamsulosin is the persistence of higher propensity in dosedependent cases. However, those at higher risk are unlikely to have received more [7]. According to Frankel, this dementia change may be a consequence of the masking of the initial symptoms, which would already be present in the course of the disease. However, if this were true, it would not be possible to evidence moderate and severe cases, as documented in the same study, and there would be no dose-dependence [4].

\section{Tamsulosin/Dose Response Ratio}

\section{Dementia Risk}

The risks of dementia associated with the tamsulosin, doxazosin, terazosin, and dutasteride groups were not different from the 
reference levels found previously or when used at low doses [7]. However, the use of tamsulosin considerably increased the risk of dementia associated with medium and high doses, which was also observed with alfuzosin and finasteride. Moreover, age or race did not change; in proportion, the association between tamsulosin use and risk of dementia in almost all analyzes $[7,8]$.

\section{Dementia and Tamsulosin}

\section{Opposing Factors}

According to Ulrich, the prescription of tamsulosin occurs in large proportions for HPB due to the simplicity of its use. Unlike other alpha-blockers, only one daily dose is required with fewer adverse effects related to orthostatic hypotension and rapid symptom improvement, reasonable efficacy, and price $[3,4]$. Importantly, the common side effects of all drugs evaluated are vasodilation, dizziness, flaccid iris syndrome, runny nose. In particular, tamsulosin may delay ejaculation in $38 \%$ of cases $[4,8]$. Another confounding factor is that tamsulosin has reduced passage in the blood-brain barrier, and it is not known how dementia can be caused even with this minimal penetration [7]. Ulrich described that this association is uncertain, because the prescription of this drug may be performed in patients who already have Mild Cognitive Impairment (MCI), which is a predictor of dementia, even if still controversial since studies are conducted a form of a retrospective analysis of medical records [3]. Another different factor is regarding the onset of dementia due to the alpha-receptor blockade. Therapeutic doses of tamsulosin, as well as drugs of the same class, could cause inhibition of adrenoreceptors. However, as evidenced by Duan et al., tamsulosin has a powerful effect [7].

According to Madersbacher and Michel, Duan's study has limitations regarding the relationship between tamsulosin use and cognitive dysfunction due to the average observation time adopted, considered relatively short (19.8 months) for a chronic pathology $[7,8]$. Another point concerns the probable selection bias since the sample selected by Duan had a higher risk of dementia at the time of tamsulosin prescription since they were elderly patients [7-9]. Kolisov et al. conducted a study by administering high doses of solifenacin $\AA$, an antimuscarinic, simultaneously to tamsulosin. There were no sequelae in the cognitive abilities of patients with BPH and symptoms of overactive bladder, and there was even an improvement in the quality of life in the experimental group $[9,10]$ In this case, the administration of higher doses of tamsulosinassociated solifenacin was effective and safe in treating BPH [8]. An alternative to quantifying and complementing the study related to the use of tamsulosin and penetration into the central nervous system causing dementia is to structure research that quantifies the Quantitative-Structure-Property Relationship (RQEP) by chromatography. RQEP models have already been tested in the use of imidazoline ligands and adrenergic receptors, with scientifically validated significance and efficiency [11].

\section{Conclusion}

In conclusion, tamsulosin is effective in treating BPH, as well as having satisfactory cost-effectiveness. However, some hypotheses need to be proven, as cognitive dysfunction may be an immediate adverse effect. Such phenomenon requires caution due to the affinity of tamsulosin with alpha-adrenergic receptors and the genesis of progressive cortical dementia. However, although cases of the association between its use and the development of dementia are described, a more in-depth study on this relationship is necessary, as other factors may be responsible for tamsulosin. As described in the discussion, some patients already had MCI which may predict increased risk for dementia, in addition to their characteristics of tamsulosin, for example, the fact that it does not cross the blood-brain barrier easily. More effort is needed in randomized experimental studies testing tamsulosin in vitro and in vivo to assess its neurotoxicity and bioavailability, especially in the central nervous system. Besides, brain biopsy studies are needed to analyze pathological changes caused by its use compared to other alpha-adrenergic antagonists and control groups.

\section{Acknowledgments}

The authors thank Prof. Dr. Aldo Medeiros, Full Professor of the Department of Surgery - UFRN, for the supervision of this review, acting as an expert consultant on the bibliographic survey, analysis, and scientific advice. We also thank all the study components for their dedication and effort to build a scientifically validated quality review.

\section{Disclosure of conflict of interest}

There are no conflicts of interest to declare by any of the authors of this study.

\section{Reference}

1. Egan KB (2016) The Epidemiology of Benign Prostatic Hyperplasia Associated with Lower Urinary Tract Symptoms: Prevalence and Incident Rates. Urol Clin North Am 43(3): 289-297.

2. Chughtai B, Forde JC, Thomas DD, Laor L, Hossack T, et al. (2016) Benign prostatic hyperplasia. Nat Rev Dis Primers. 2: 16031.

3. Ulrich S (2018) Differential prescription behavior in benign prostatic syndrome may explain relationship found between tamsulosin and dementia. Pharmacoepidemiol Drug Saf 27(11): 1157-1158.

4. Frankel JK, Duan Y, Albertsen PC (2018) Is Tamsulosin Linked to Dementia in the Elderly? Curr Urol Rep 19(9): 69.

5. Roehrborn CG, Oyarzabal Perez I, Roos EP, Calomfirescu N, Brotherton B, et al. (2015) Efficacy and safety of a fixed-dose combination of dutasteride and tamsulosin treatment (Duodart $(囚)$ ) compared with watchful waiting with initiation of tamsulosin therapy if symptoms do not improve, both provided with lifestyle advice, in the management of treatment-naïve men with moderately symptomatic benign prostatic hyperplasia: 2-year CONDUCT study results. BJU Int 116(3): 450-459.

6. Gill SS, Bai AD (2018) Beta testing the potential link between the alpha antagonist tamsulosin and dementia. Pharmacoepidemiol Drug Saf 27(3): 349-350.

7. Madersbacher S, Michel MC (2018) Re: Tamsulosin and the Risk of Dementia in Older Men with Benign Prostatic Hyperplasia. Eur Urol. 74(4): 522-523. 
8. Kosilov K, Kuzina I, Kuznetsov V, Gainullina Y, Kosilova L, et al. (2018) Cognitive functions and health-related quality of life in men with benign prostatic hyperplasia and symptoms of overactive bladder when treated with a combination of tamsulosin and solifenacin in a higher dosage. Aging Male 21(2): 121-129.

9. Nikolic K, Filipic S, Smoliński A, Kaliszan R, Agbaba D (2013) Partial least square and hierarchical clustering in ADMET modeling: prediction of blood-brain barrier permeation of $\alpha$-adrenergic and imidazoline receptor ligands. J Pharm Pharm Sci 16(4): 622-647.
10. Duan Y, Grady JJ, Albertsen PC, Helen Wu Z (2018) Tamsulosin and the risk of dementia in older men with benign prostatic hyperplasia. Pharmacoepidemiol Drug Saf 27(3): 340-348.

11. Szot P, White SS, Greenup JL, Leverenz JB, Peskind ER, et al. (2007) Changes in adrenoreceptors in the prefrontal cortex of subjects with dementia: evidence of compensatory changes. Neuroscience 146(1): 471-80. 